\title{
Lösliche Serumfaktoren als prognostische Marker des Melanoms: sHLA, sFas und IL-8
}

\author{
S. Ugurel ${ }^{1}$ \\ W. Tilgen ${ }^{2}$ \\ U. Reinhold ${ }^{2}$
}

\author{
Soluble Factors as Prognostic Serological Markers in Malignant Melanoma: \\ SHLA, sFas and IL-8
}

\section{Zusammenfassung}

Eine größere Anzahl serologischer Faktoren konnte in den letzten Jahren als Tumormarker des malignen Melanoms identifiziert werden. Für einen Großteil dieser Markerproteine konnte eine Korrelation mit der Prognose der Patienten gezeigt werden. Trotzdem gibt es aktuell keine internationalen Empfehlungen hinsichtlich eines routinemäßigen Einsatzes eines dieser Markerproteine. Der aktuell am häufigsten eingesetzte Marker ist das S100- $\beta$ Protein, das ursprünglich als saures, Kalzium-bindendes Protein aus Rinderhirnextrakt dargestellt wurde. S100- $\beta$ zeigte sich als nützlicher prognostischer Serummarker bei Patienten mit Melanom im metastasierten Stadium und stellt einen guten Verlaufsparameter für das Monitoring metastasierter Melanompatienten unter Chemotherapie dar. Andere Serumproteine, wie z.B. Melanoma Inhibiting Activity (MIA), wurden ebenfalls in zahlreichen Studien als serologische Prognoseindikatoren des Melanoms evaluiert, konnten jedoch die Aussagekraft des S100- $\beta$ nicht übertreffen. Ein bedeutender Nachteil des S100- $\beta$ ist dessen geringer prädiktiver Wert hinsichtlich der Prognose bei Patienten in frühen Erkrankungsstadien, vor Nachweis einer Metastasierung. Diese Patienten werden nach Exzision des Primärtumors aufwändigen Nachsorge-Untersuchungsprogrammen unterzogen, um eine eventuell auftretende Metastasierung möglichst frühzeitig zu erkennen und zu behandeln. Hierzu müssen diese Patienten aktuell invasive und kostenintensive Maßnahmen, wie beispielsweise die Exstirpation des Sentinel Lymphknotens, in Kauf nehmen. Insbesondere diese Patientengruppe würde von der Entwicklung neuer sensitiver und hinreichend genauer prognostischer Marker, die wenig aufwändig aus dem Serum des Patienten bestimmt werden könnten, profitieren.

\section{Abstract}

A variety of serological markers have been identified in malignant melanoma, most of them associated with disease progression. Nevertheless, none of them is currently recommended for routine diagnostic use by international guidelines. S100- $\beta$, originally described as an acidic, calcium-binding protein derived from a bovine brain extract, has been demonstrated as an appropriate serum marker in metastatic melanoma patients with high serum levels indicating disease progression or recurrence. Moreover, $\mathrm{S} 100-\beta$ has been proved as a useful serum parameter in the monitoring of metastatic melanoma patients during ongoing chemotherapy. Other serological marker proteins like e.g. melanoma inhibiting activity (MIA) have been evaluated in several studies, but could not be approved to exceed the predictive value of S100- $\beta$. A major disadvantage of S100- $\beta$ is the poor predictive value of this marker in early-stage non-metastasized melanoma patients. After surgical removal of their primary tumors, these patients are enrolled into elaborated follow-up examination programs aiming at a detection of recurrence of the disease as early as possible. To estimate their risk of recurrence, these patients currently have to undergo invasive and costly procedures like e.g. sentinel lymph node dissection. Therefore, the identification of sensitive and reliable serological markers with strong predictive impact for the patients' prognosis would be of high beneficial value for this group of patients.

${ }^{1}$ Klinische Kooperationseinheit Dermato-Onkologie (Leiter: Prof. D. Schadendorf),

Deutsches Krebsforschungszentrum Heidelberg/Klinik für Dermatologie, Venerologie und Allergologie,

Universitätsklinikum Mannheim

${ }^{2}$ Universitäts-Hautklinik und Poliklinik (Direktor: Prof. W. Tilgen), Universitätskliniken

des Saarlandes, Homburg/Saar

Korrespondenzadresse

Selma Ugurel · Klinische Kooperationseinheit für Dermato-Onkologie (DKFZ) ·

Universitätsklinikum Mannheim · Theodor-Kutzer-Ufer 1 . 68135 Mannheim 
Die Inzidenz des malignen Melanoms ist weltweit steigend [1]. Trotz intensiver Bemühungen in der Entwicklung neuer Therapieansätze sind die Erfolge in der Behandlung des metastasierten Melanoms bis heute eher als eingeschränkt zu werten. Umso mehr erscheint es wichtig, Patienten mit erhöhter Wahrscheinlichkeit einer Erkrankungsprogression im Sinne einer lymphogenen oder hämatogenen Metastasierung zu einem möglichst frühen Zeitpunkt zu identifizieren. Diese Patienten könnten in der Folge einer engmaschigeren klinischen oder apparativen Nachsorge-Diagnostik bzw. adäquaten therapeutischen Maßnahmen unterzogen werden. Es besteht somit die Notwendigkeit, für das maligne Melanom nicht „Tumormarker“ im Allgemeinen, sondern „prognostische Marker“ im Speziellen zu identifizieren und in der Folge hinsichtlich ihrer Wertigkeit und prädiktiven Aussagekraft zu evaluieren. Diese Marker müssten somit nicht zwangsläufig spezifisch für die Tumorentität Melanom sein, da sie nicht zur Diagnosefindung oder zur Verlaufskontrolle, sondern zur Einschätzung des progressionsfreien und des Gesamtüberlebens des Patienten zu einem definierten Zeitpunkt eingesetzt werden sollten. Als Haupteinsatzgebiet derartiger Marker käme die Einschätzung der Prognose von Melanompatienten nach operativ erreichter Tumorfreiheit, insbesondere in frühen Erkrankungsstadien (AJCC I/II), aber auch nach bereits erfolgter Metastasierung (Stadium III/IV) in Betracht. Eine unlängst entwickelte Methode zur Prädiktion der Prognose bei Melanompatienten ist die so genannte „Sentinel Lymph Node Dissection“, die diagnostische Exstirpation des Schildwächter-Lymphknotens und nachfolgende sowohl konventionell histologische als auch immunhistologische Untersuchung des Exzidats. Es zeigte sich eine deutlich ungünstigere Prognose bei Patienten mit positivem Nachweis einer Mikrometastasierung des Sentinel Lymphknotens verglichen mit Patienten mit negativer Histologie [2]. Allerdings ist dieses Verfahren aufwändig und für den Patienten mit operativ-invasiven Maßnahmen verbunden. Einfacher, weniger invasiv und nicht zuletzt auch kostengünstiger wäre eine Prognoseeinschätzung mittels sensitiver löslicher Faktoren in Serum oder Plasma der Patienten. Eine derartige Untersuchung ließe sich auch beliebig oft zu späteren Zeitpunkten wiederholen.

Tab. 1 Prognostische Marker des malignen Melanoms: sHLA, sFas/CD95, IL-8

\begin{tabular}{|c|c|c|c|c|c|c|c|}
\hline Marker & Literaturstelle & $\begin{array}{l}\text { untersuchtes } \\
\text { Medium }\end{array}$ & $\begin{array}{l}\text { Anzahl der } \\
\text { Patienten }\end{array}$ & $\begin{array}{l}\text { Stadium } \\
\text { (AJCC) }\end{array}$ & $\begin{array}{l}\text { Anzahl der } \\
\text { Kontrollen }\end{array}$ & $\begin{array}{l}\text { Korrelation mit } \\
\text { Gesamtüberleben }\end{array}$ & $\begin{array}{l}\text { Korrelation mit } \\
\text { progressionsfreiem Überleben }\end{array}$ \\
\hline sHLA-DR & Rebmann et al. (2002) [15] & Serum & 183 & $1 / 11 / 111 / / \mathrm{IV}$ & 86 & $+(p=0,021)$ & $+(p<0,0005)$ \\
\hline sHLA-I & Westhoff et al. (1998) [17] & Plasma & 105 & I/II/III/IV & 55 & n.t. & $+(p=0,04)$ \\
\hline sHLA-G & Ugurel et al. (2001) [22] & Serum & 190 & I/IIIIIII/IV & 126 & $-(p>0,05)$ & $-(p>0,05)$ \\
\hline \multirow[t]{2}{*}{ sFas/CD95 } & Mouawad et al. (2000) [45] & Plasma & 45 & IV & 34 & n.t. & n.t. \\
\hline & Ugurel et al. (2001) [46] & Serum & 125 & I/II/III/IV & 30 & $+(p<0,005)$ & $+(p<0,0005)$ \\
\hline \multirow[t]{2}{*}{ IL-8 } & Scheibenbogen et al. (1995) [55] & Serum & 56 & IV & 30 & n.t. & n.t. \\
\hline & Ugurel et al. (2001) [56] & Serum & 125 & I/II/III/IV & 30 & $+(p<0,00005)$ & $+(p<0,00005)$ \\
\hline
\end{tabular}

Alle Untersuchungen wurden mittels ELISA-Technik durchgeführt; n.t.: nicht getestet
Verschiedenste lösliche Moleküle wurden bereits hinsichtlich ihrer prognostischen Relevanz beim malignen Melanom untersucht und beschrieben (siehe Tab.1). Als bekanntester und bereits als weitgehend etabliert einzustufender Marker ist das S100- $\beta$-Protein zu nennen. Die Serumkonzentration des S100- $\beta$ wurde in verschiedenen Arbeiten als guter Indikator sowohl der Prognose [3,4] als auch des Therapieverlaufes [5] bei Melanompatienten beschrieben. Ein Schwachpunkt des S100- $\beta$ als prognostischer Marker ist die geringe prädiktive Wertigkeit bei Patienten in frühen Erkrankungsstadien (AJCC I/II) [3,4]. Als weiterer ausführlich untersuchter Serummarker mit einer guten Korrelation zur Prognose kann das Melanoma Inhibitory Activity Protein (MIA) angesehen werden [6]. Im Gegensatz zu S100- $\beta$ konnten hier auch bei Patienten in frühen Erkrankungsstadien vor erfolgter Metastasierung bereits erhöhte Serumwerte für MIA gefunden werden (13\% in AJCC Stadium I, 23\% in AJCC Stadium II), die mit einer erhöhten Progressionswahrscheinlichkeit dieser Patienten assoziiert waren [6]. Weitere Untersuchungen zeigten jedoch einen weitaus niedrigeren Anteil an Patienten mit erhöhten MIA-Serumwerten in einer vergleichbaren Patientengruppe (5,6\% in AJCC Stadium I/II) [7]. Ein nicht melanomspezifischer, jedoch zur Vorhersage der Prognose von fernmetastasierten Melanompatienten (AJCC Stadium IV) gut geeigneter Serumfaktor ist die Laktat-Dehydrogenase (LDH) [8]. Dieser Marker zeigt eine starke Korrelation zur Tumorlast, ist jedoch zur Prognoseeinschätzung bei tumorfreien Patienten wenig geeignet.

\section{Lösliche HLA-Moleküle als prognostische Serummarker}

Human leukocyte antigen(HLA)-Moleküle sind von herausragender Wichtigkeit für die Interaktion zwischen Tumorzellen und Effektorzellen des Immunsystems [9]. Tumorassoziierte Antigene, deren Erkennung durch antigenspezifische T-Lymphozyten essenziell für den Ablauf sowohl der humoralen als auch der zellulären antitumoralen Immunantwort ist, werden an der Oberfläche der Tumorzellen durch HLA-Moleküle präsentiert. Des Weiteren ist die Expression von HLA-Molekülen auf den verschiedenen lymphomonozytären Zelltypen von ebenso großer Bedeutung für die Immunabwehr der Tumorzellen, hier beispielsweise auf der Oberfläche professioneller antigenpräsentierender Zellen. 


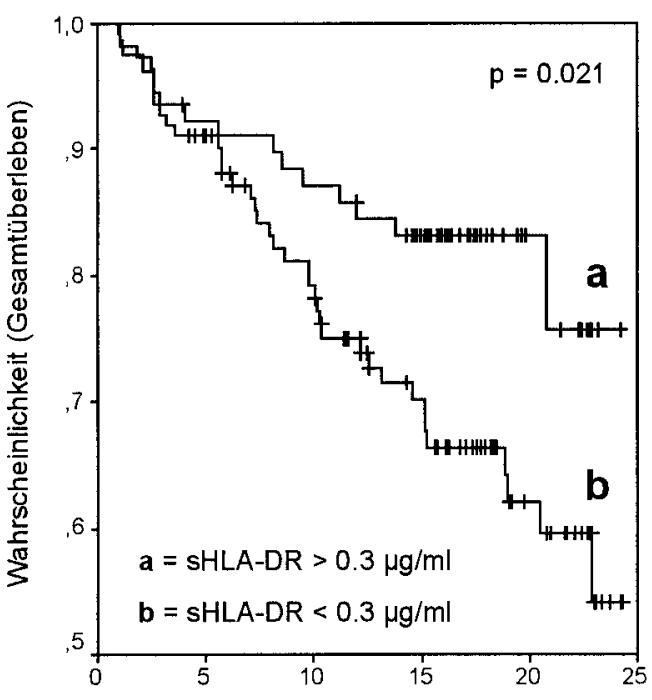

a

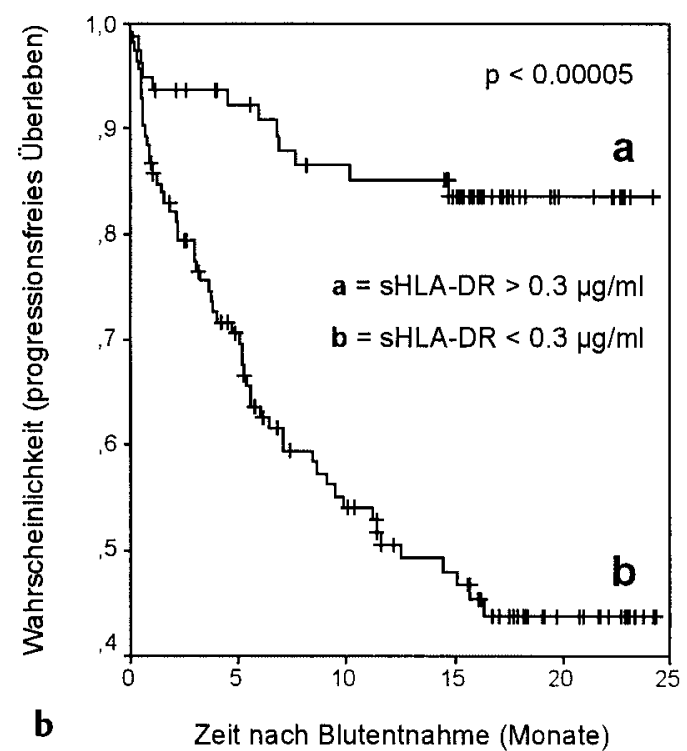

Zeit nach Blutentnahme (Monate)

Abb. 1 sHLA-DR als Serummarker mit prädiktiver Wertigkeit für das progressionsfreie und das Gesamtüberleben von Melanompatienten. Kaplan-Meier-Kurven zur Darstellung des Gesamtüberlebens (a) und des progressionsfreien Überlebens (b) bei 183 Melanompatienten. Die Patienten wurden nach ihren Serumwerten für sHLADR in 2 Gruppen unterteilt ( $\mathrm{a}=75$ Patienten, $\mathrm{b}=108$ Patienten); der verwendete Cut-off-Wert von 0,3 $\mu \mathrm{g} / \mathrm{ml}$ wurde mittels ROC-Kurvenanalyse bestimmt. Die statistischen Signifikanzen, angegeben in der rechten oberen Bildecke, wurden mittels des Log-Rank-Tests bestimmt. Modifiziert aus: Rebmann et al. 1) [15].

Lösliche Formen der HLA-Moleküle konnten bereits vor längerer Zeit im Serum und in anderen Körperflüssigkeiten gefunden werden [10,11]. Diese löslichen HLA-Moleküle (sHLA) entstehen durch Abscherung membranständiger HLA-Moleküle von der Zelloberfläche (Shedding) oder durch zelluläre Sekretion und sind in der Lage, an die gleichen Liganden wie ihre membranständigen Dependants zu binden und über diese Bindung verschiedene Effekte, wie z.B. die Induktion einer Apoptose, auszuüben [12]. Lösliche HLA-Moleküle könnten somit im Serum sowohl ein Abbild der HLA-Zelloberflächen-Expression des Patienten darstellen als auch selbst immunmodulatorische Funktionen ausüben.

Lösliche HLA-Klasse-II-Antigene HLA-DR (sHLA-DR) konnten kürzlich als Serum-Indikator für den Status der Immun-Aktivierung eines Patienten beschrieben werden. So zeigten beispielsweise Patienten unter aktiver Virus-Infektion oder Graft-VersusHost-Disease erhöhte Serum-Konzentrationen für sHLA-DR [13]. Dagegen wiesen Patienten mit reduzierter Immunabwehrfunktion, z. B. unter schwerer Sepsis, deutlich erniedrigte Serumwerte für sHLA-DR auf [14]. In einer größeren Untersuchung an 183 Melanompatienten unterschiedlicher Erkrankungsstadien fanden sich deutlich erniedrigte sHLA-DR-Serumwerte bei Patienten mit reduzierter Prognose (Abb.1) [15]. Im Gegensatz zu den gleichzeitig bei diesen Patienten gemessenen Serumwerten für S100- $\beta$ fand sich insbesondere auch bei Patienten in frühen Erkrankungsstadien (AJCC I/II) eine starke Assoziation erniedrigter sHLA-DR-Serumkonzentrationen mit einer ungünstigen Prognose (Abb. 2). sHLA-DR konnte in diesem Zusammenhang als unabhängiger prognostischer Faktor des progressionsfreien Überlebens bei Melanompatienten gezeigt werden (Abb.3). Vermutet und aktuell untersucht wird eine Funktion des sHLA-DR als Abbild einer erniedrigten Expression von HLA-DR-Molekülen auf Effektorzellen des Immunsystems und somit als Indikator einer verminderten Funktion der Antigen-Präsentation im Rahmen der antitumoralen Immunabwehr.

HLA-Klasse-I-Moleküle spielen eine bedeutende Rolle im Rahmen der antitumoralen Immunabwehr durch ihre antigenpräsentierende Funktion gegenüber zytotoxischen T-Lymphozyten. Ein kompletter oder teilweiser Verlust der HLA-Klasse-I-Expression auf malignen Zellen führt zu einer verminderten spezifischen Erkennung und Zerstörung dieser Zellen durch die Effektorzellen des Immunsystems [16]. Lösliche Formen klassischer HLA-Klasse-I-Moleküle (sHLA-I) wurden kürzlich in Plasmen von 105 Melanompatienten untersucht [17]. Hierbei ergab sich eine Assoziation erniedrigter Plasmawerte für sHLA-I mit erhöhter Tumoreindringtiefe und Metastasierung, jedoch nur eine schwache Korrelation zur Prognose der Patienten. Weitere Untersuchungen an größeren Patientenkollektiven sind in Planung, um die prädiktive Wertigkeit von sHLA-I bei Melanompatienten zu evaluieren.

Das nicht-klassische HLA-Klasse-I-Molekül HLA-G wurde ursprünglich als ausschließlich auf fetalen Zellen der Plazenta exprimiertes Protein beschrieben und hat eine zentrale Bedeutung für die Aufrechterhaltung einer immunprivilegierten Zone an der maternofetalen Grenzregion [18,19]. Auch maligne entartete Zellen schaffen sich durch verschiedene Escape-Mechanismen ein immunprivilegiertes Umgebungsmilieu, so z.B. durch eine verminderte oder gänzlich fehlende Expression klassischer HLAKlasse-I-Moleküle [16]. Hierdurch bieten diese Zellen wiederum einen Angriffspunkt für natürliche Killerzellen (NK-Zellen). Eine Expression von HLA-G an der Zelloberfläche würde die Tumorzellen vor einem NK-Zell-Angriff bewahren. So konnte kürzlich eine HLA-G-Expression für Melanomzellen nachgewiesen werden, die diese Zellen effektiv vor einer Lyse durch NK-Zellen schützte [20]. Lösliche HLA-G-Moleküle (sHLA-G) konnten be- 

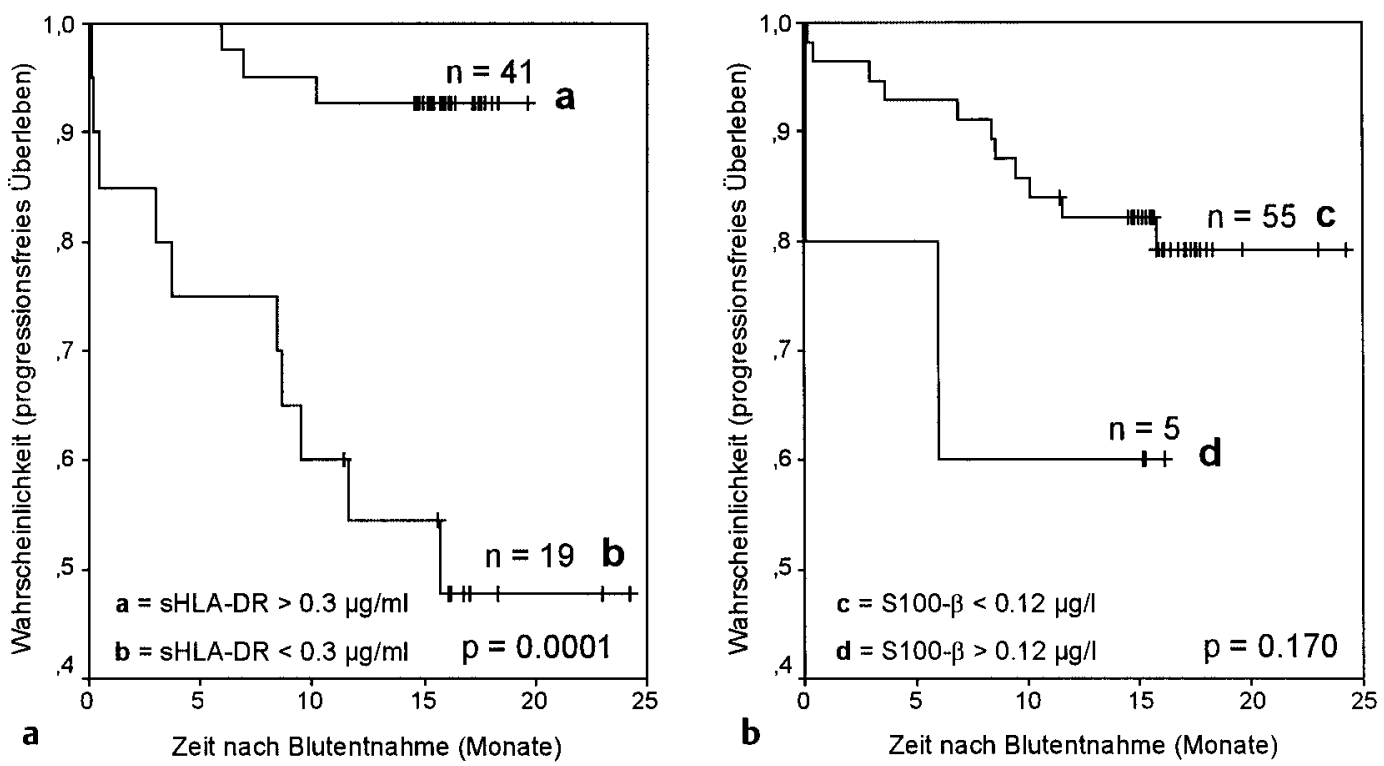

Abb. 2 sHLA-DR ermöglicht eine bessere prognostische Einschätzung des progressionsfreien Überlebens von AJCC-Stadium I/II Melanompatienten als S100- $\beta$. Kaplan-Meier-Kurven zur Darstellung des progressionsfreien Überlebens bei 60 Melanompatienten in frühen Erkrankungsstadien (AJCC I/II). Die Patienten wurden anhand ihrer Serumwerte für SHLA-DR (a) und S100- $\beta$ (b) in 2 Gruppen unterteilt; der verwendete Cut-off-Wert von $0,3 \mu \mathrm{g} / \mathrm{ml}$ für sHLA-DR wurde mittels ROC-Kurvenanalyse bestimmt (siehe Abb. 1), für S100- $\beta$ wurde der vom Hersteller angegebene Cut-off-Wert von $0,12 \mu \mathrm{g} / \mathrm{l}$ verwendet. Die statistischen Signifikanzen, angegeben in der rechten unteren Bildecke, wurden mittels des Log-Rank-Tests bestimmt. Modifiziert aus: Rebmann et al. [15].

ROC-Kurve

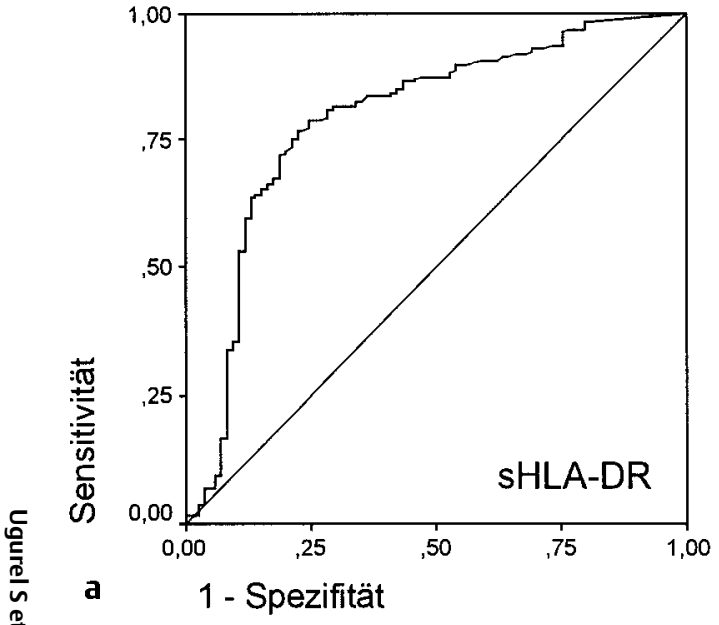

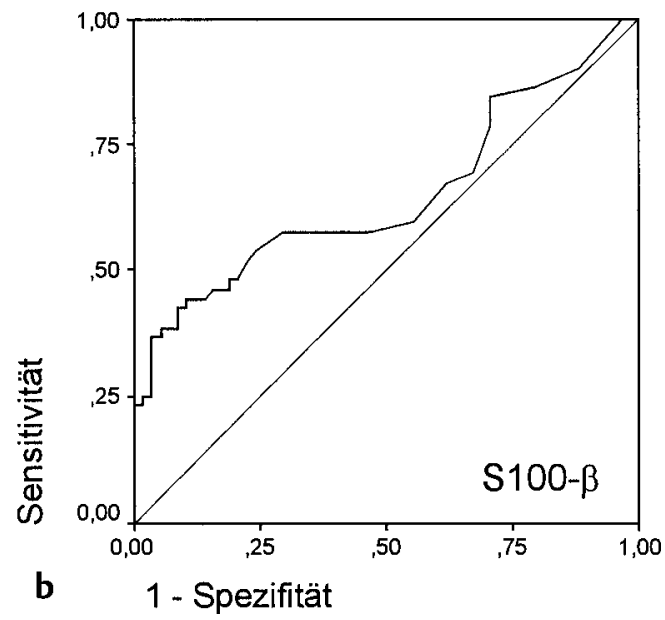

Abb. 3 sHLA-DR hat eine höhere prognostische Aussagekraft hinsichtlich des progressionsfreien Überlebens von Melanompatienten als S100- $\beta$. Receiver operating characteristic (ROC) Kurvenanalyse zur Sensitivität und Spezifität von sHLA-DR (a) im Vergleich zu S100- $\beta$ (b) hinsichtlich der prädiktiven Wertigkeit für das progressionsfreie Überleben bei 183 Melanompatienten. Als optimaler Cut-off-Wert wurde für sHLA-DR 0,3 $\mu \mathrm{g} / \mathrm{ml}$ bestimmt, für S100- $\beta$ konnte kein Cut-off-Wert berechnet werden. Modifiziert aus: Rebmann et al. [15].

reits im Serum sowie in verschiedenen anderen Körperflüssigkeiten nachgewiesen werden [21]. In einer größeren Studie an 190 Melanompatienten unterschiedlicher Erkrankungsstadien fanden sich zwar erhöhte Serumwerte für sHLA-G in der Patientengruppe im Vergleich zur Kontroll-Gruppe sowie ein starker Einfluss einer systemischen Therapie mit Interferon(IFN)- $\alpha$ auf die sHLA-G-Serumkonzentration (Abb.4a), jedoch keine Korrelation zur Überlebenswahrscheinlichkeit (Abb. 4b) [22]. sHLA-G ist somit keine potenzielle Funktion als prädiktiver Serummarker beim malignen Melanom zuzuordnen.
Lösliches Fas/CD95 (sFas) als prognostischer Serummarker Apoptose-Mechanismen, deren Ziel der so genannte „programmierte Tod" der Zelle darstellt, spielen eine bedeutende Rolle für die Proliferation und Immunabwehr maligner Tumoren [23]. Sowohl die Effizienz der zellulären antitumoralen Immunantwort als auch die Wirksamkeit zahlreicher Zytostatika basieren essenziell auf einer hinreichenden Funktion der die Apoptose auslösenden Signalwege. Das Fas/CD95-FasLigand(FasL)-System spielt in diesem Zusammenhang eine entscheidende Rolle als Angriffspunkt der zellulären Immunabwehr, z.B. der zytotoxi- 


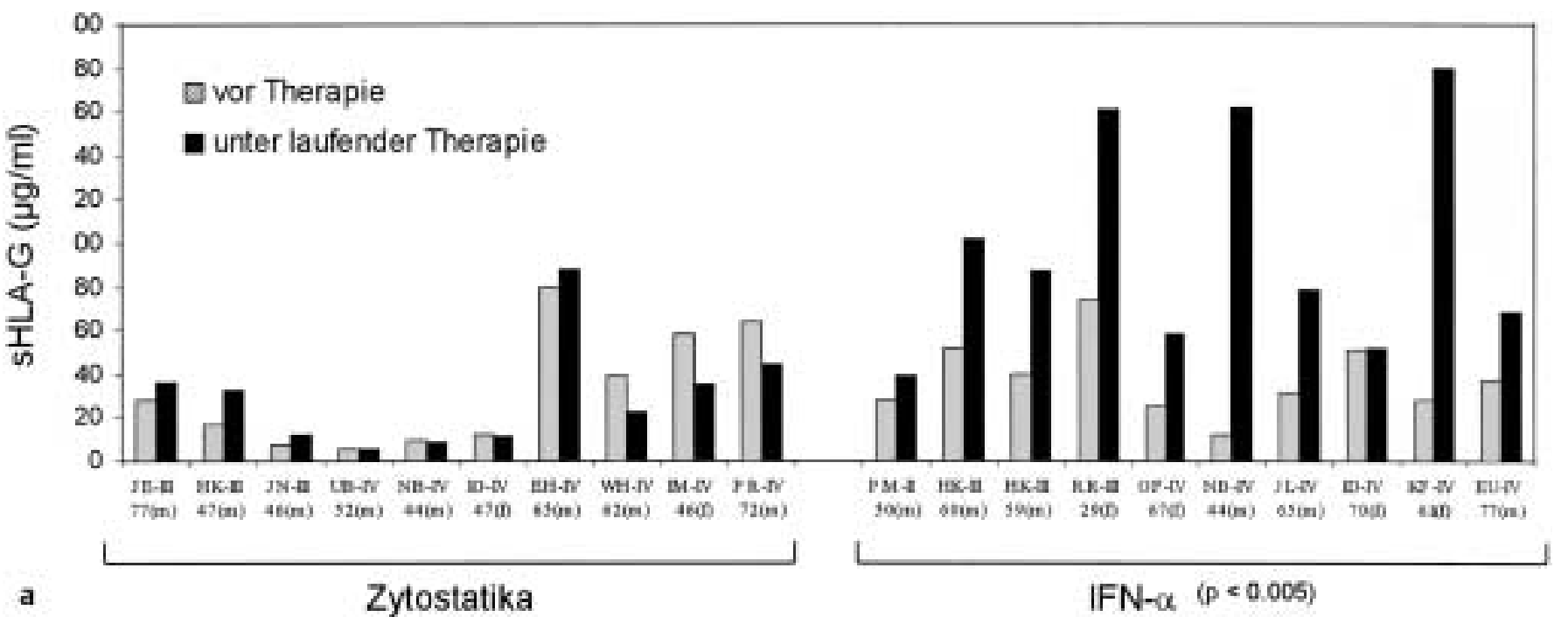

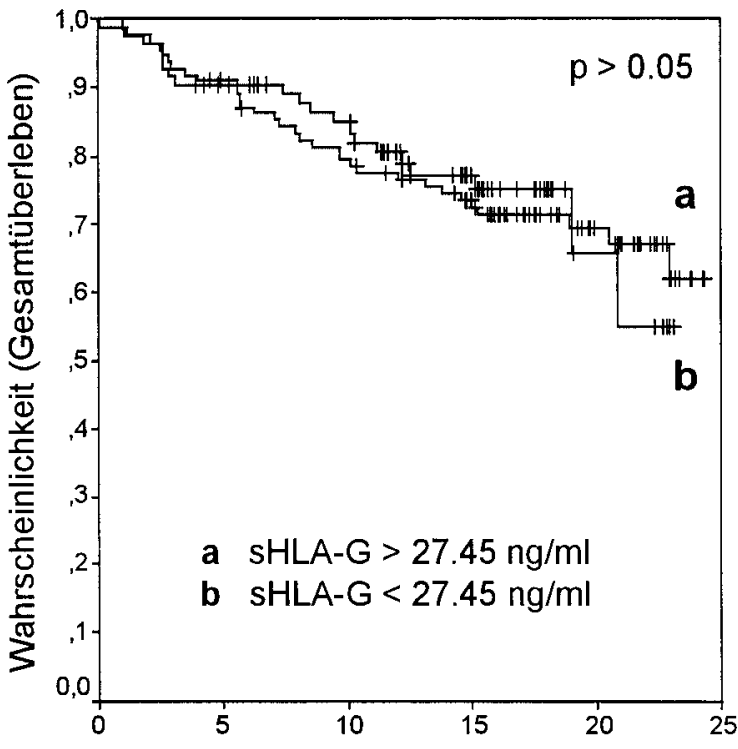

b Zeit nach Blutentnahme (Monate)

schen CD8 ${ }^{+}$T-Lymphozyten [24]. Fas/CD95 ist ein membranständiges Rezeptormolekül, das zu der Familie der so genannten Todesrezeptoren gehört. Durch Bindung des FasL-Moleküls wird eine Quervernetzung mehrerer Fas/CD95-Rezeptormoleküle hervorgerufen, die eine Signaltransduktion in das Zellinnere auslöst, in deren Folge es durch Ablauf einer enzymatischen Kaskade zum Tod der Zelle kommt [23] (Abb.5). Für Tumorzellen unterschiedlicher Herkunft wurde eine verminderte Expression oder gestörte Funktion der Fas/CD95-Expression beschrieben [25, 26]. Bezogen auf das maligne Melanom konnte eine besonders stark ausgeprägte Dysfunktion des Fas/CD95-FasL-Systems nachgewiesen werden [27-31], für die eine wichtige Bedeutung für den Metastasierungsprozess gezeigt werden konnte [32]. Wie auch für andere Rezeptormoleküle bekannt existieren auch im Falle des Fas/CD95 lösliche Molekülformen, denen der transmembranäre Anteil fehlt [33]. Diese entstehen jedoch im Falle des Fas/CD95 im Gegensatz zu anderen Rezeptormolekülen nicht durch Abscherung membranständiger Moleküle von der Zelloberfläche (Shedding), sondern durch alternatives Splicing und
Abb. 4 Erhöhte sHLA-G-Serumspiegel bei Melanompatienten werden durch Therapie mit IFN- $\alpha$ induziert und sind ohne prognostische Relevanz. (a) sHLA-G-Serumkonzentrationen aus jeweils 10 repräsentativen Melanompatienten vor und unter laufender Chemotherapie bzw. IFN- $\alpha$ Immuntherapie. $\mathrm{p}<0,005$ für Differenzen vor/unter Therapie mit IFN- $\alpha$, Student's t-Test für gepaarte Stichproben. (b) Kaplan-Meier-Kurven zur Darstellung des Gesamtüberlebens bei 190 Melanompatienten. Die Patienten wurden nach ihren Serumwerten für sHLA-G in 2 Gruppen unterteilt; der verwendete Cut-off-Wert von $27,45 \mathrm{ng} / \mathrm{ml}$ wurde mittels ROC-Kurvenanalyse bestimmt. Die statistische Signifikanz, angegeben in der rechten oberen Bildecke, wurde mittels des Log-Rank-Tests bestimmt. Modifiziert aus: Ugurel et al. [22].

anschließende Sekretion durch die Zelle [34]. Diese löslichen Fas/CD95 Moleküle (sFas) sind in der Lage, durch Bindung an FasL Moleküle diese für eine weitere Bindung an membranständige Fas/CD95-Rezeptormoleküle zu blockieren [33] (Abb. 5). Die FasL tragenden Effektorzellen, z. B. CD8 ${ }^{+}$-zytotoxische T-Lymphozyten, sind somit hinsichtlich ihrer Fähigkeit, in Fas/CD95-tragenden Zielzellen eine Apoptose auszulösen, deutlich eingeschränkt. Erhöhte Serumwerte für sFas konnten erstmals bei Patienten mit aktiven Autoimmunerkrankungen nachgewiesen werden [35]. Wenig später wurden bei Patienten mit malignen Erkrankungen ebenfalls erhöhte Serumwerte für sFas beschrieben [36 - 44], die in einigen Fällen eine Korrelation mit der Prognose der Patienten aufwiesen [36,39,40,42-44]. Innerhalb eines größeren Kollektivs von Tumorpatienten mit unterschiedlichen Neoplasien wurden erstmals 8 Patienten mit malignem Melanom untersucht, die im Vergleich zu gesunden KontrollProbanden erhöhte sFas-Serumkonzentrationen aufwiesen [36]. In einer weiteren Untersuchung zeigten sich erhöhte sFas-Konzentrationen in Plasma von 45 Melanompatienten im fernmetas- 


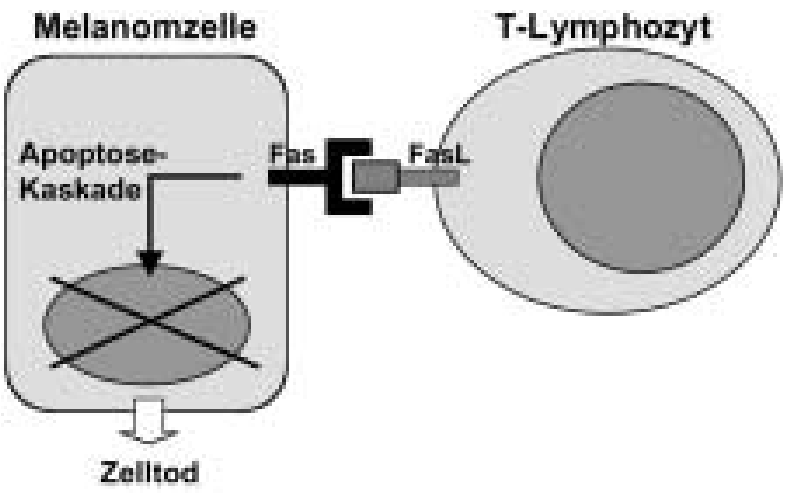

B

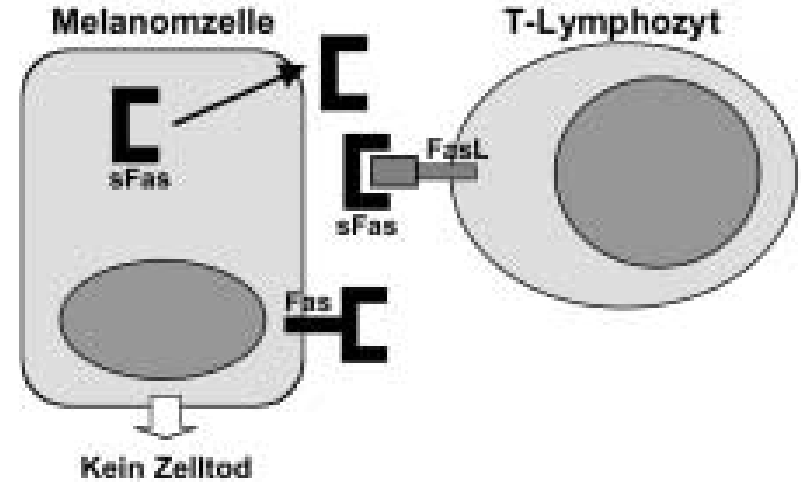

Abb. 5 Lösliche Fas-Moleküle (sFas) blockieren die Fas-vermittelte Induktion der Apoptose in Melanomzellen. (A) Fas-Ligand(FasL)-tragende zytotoxische T-Lymphozyten sind durch Bindung an den Fas-Rezeptor (Fas) auf der Oberfläche von Melanomzellen in der Lage, diese durch Auslösung einer enzymatischen Kaskade in den Tod durch Apoptose zu treiben. (B) Von Melanomzellen sezernierte lösliche Fas-Moleküle (sFas) blockieren FasL-Moleküle auf der Oberfläche zytotoxischer T-Lymphozyten und verhindern somit die Induktion einer Fas-vermittelten Apoptose in Melanomzellen. tasierten Stadium (AJCC IV) [45]. Eine größere Studie an 125 Melanompatienten unterschiedlicher Erkrankungsstadien ergab deutlich erhöhte Serumkonzentrationen für sFas bei Melanompatienten im Vergleich zu 30 untersuchten gesunden KontrollProbanden [46]. Die erhöhten Serumwerte zeigten eine starke Korrelation zu fortgeschrittenen Erkrankungsstadien, jedoch nur eine schwache Assoziation zu nachweisbarer Tumorlast der Patienten. Des Weiteren fanden sich deutlich erhöhte sFas-Serumkonzentrationen bei Patienten mit ungünstiger Prognose (Abb. 6). sFas konnte im Rahmen dieser Studie als unabhängiger prognostischer Faktor für das progressionsfreie Überleben der Patienten gezeigt werden. Weitere Untersuchungen sind geplant, insbesondere zur Analyse der Herkunft der löslichen FasMoleküle bei Patienten mit malignem Melanom.

\section{Interleukin-8 (IL-8) als prognostischer Serummarker}

Interleukin-8 (IL-8), zugehörig zur Gruppe der CXC-Chemokine, ist bekannt für seine pro-inflammatorische [47] sowie pro-angiogenetische [48] Wirkung. Bezogen auf das maligne Melanom konnte IL-8 bereits vor einiger Zeit als autokriner Wachstumsfaktor beschrieben werden [49]. Eine Rolle der IL-8-Expression für die Progression und Metastasierung des Melanoms ist wahrscheinlich [50-54]. Eine erste Untersuchung der IL-8-Serumkonzentration bei 56 Melanompatienten zeigte erhöhte Werte bei Patienten im Vergleich zu gesunden Kontroll-Probanden sowie eine Korrelation der IL-8-Serumwerte mit der Tumorlast der Patienten [55]. In einer größeren Studie an 125 Melanompatienten fand sich eine Korrelation der IL-8-Serumkonzentration zu Erkrankungsstadium und Tumorlast der Patienten [56]. Hinsichtlich der Prognose der Patienten konnte IL-8 in dieser Arbeit als unabhängiger prädiktiver Faktor sowohl für das progressionsfreie Überleben als auch für das Gesamtüberleben der Patienten dargestellt werden (Abb. 7). Erhöhte Serumwerte für IL-8 wurden hier jedoch ausschließlich in Patienten mit metastasierter Erkrankung detektiert. IL-8 stellt somit einen guten prädiktiven
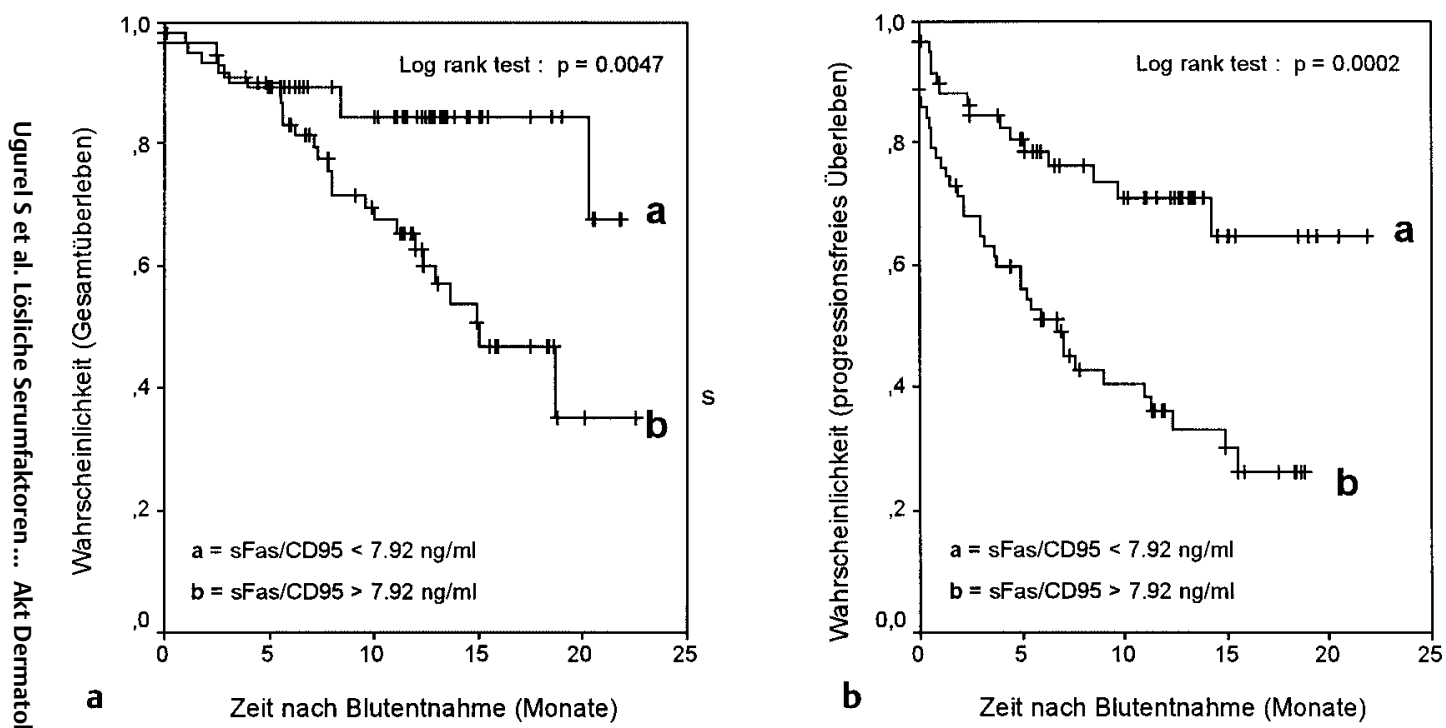

Abb. 6 sFas als Serummarker mit prädiktiver Wertigkeit für das progressionsfreie und das Gesamtüberleben von Melanompatienten. Kaplan-Meier-Kurven zur Darstellung des Gesamtüberlebens (a) und des progressionsfreien Überlebens (b) bei 125 Melanompatienten. Die Patienten wurden anhand ihrer Serumwerte für sFas in 2 Gruppen unterteilt; der verwendete Cut-off-Wert von 7,92 ng/ml wurde mittels ROC-Kurvenanalyse bestimmt. Die statistischen Signifikanzen, angegeben in der rechten oberen Bildecke, wurden mittels des Log-Rank-Tests bestimmt. Modifiziert aus: Ugurel et al. [46]. 

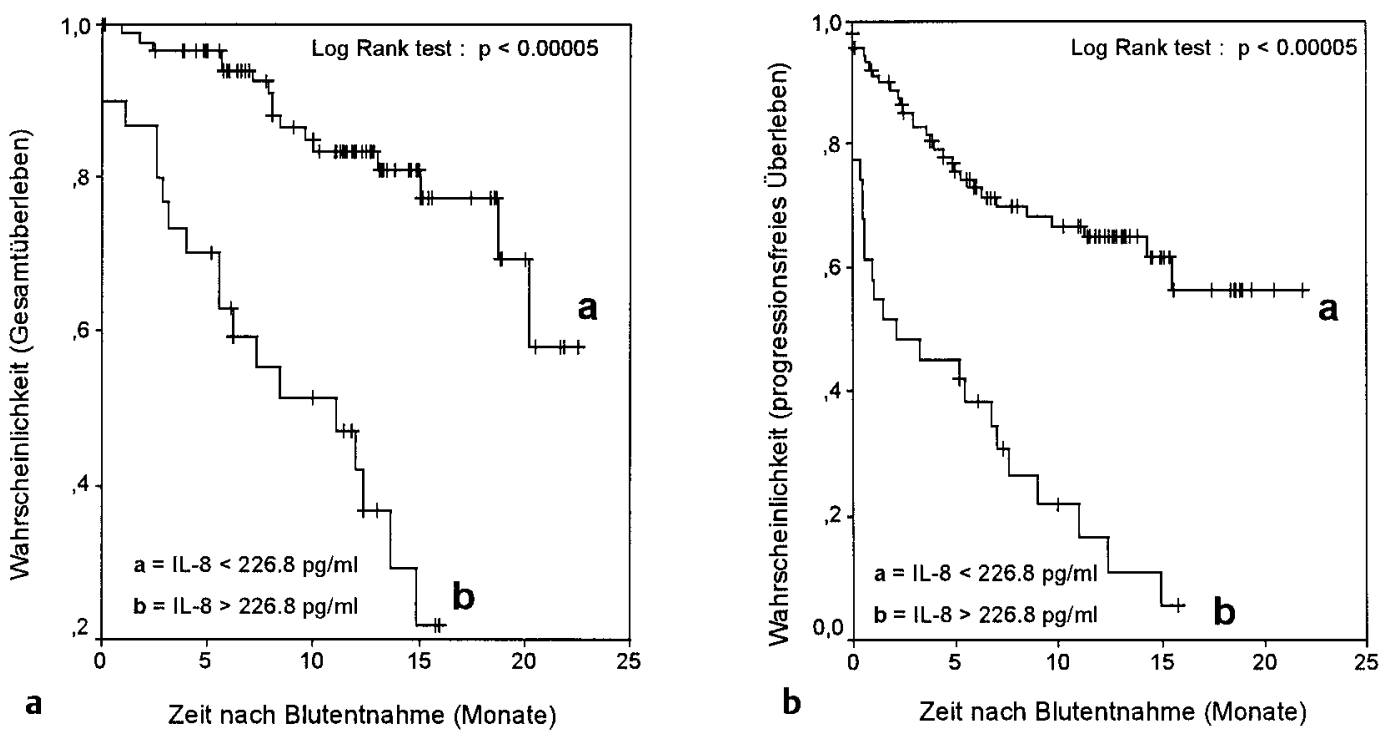

Abb. 7 Interleukin-8 (IL-8) als Serummarker mit prädiktiver Wertigkeit für das progressionsfreie und das Gesamtüberleben von Melanompatienten. Kaplan-Meier-Kurven zur Darstellung des Gesamtüberlebens (a) und des progressionsfreien Überlebens (b) bei 125 Melanompatienten. Die Patienten wurden anhand ihrer Serumwerte für IL-8 in 2 Gruppen unterteilt; der verwendete Cut-off-Wert von 226,8 pg/ml wurde mittels ROC-Kurvenanalyse bestimmt. Die statistischen Signifikanzen, angegeben in der rechten oberen Bildecke, wurden mittels des Log-Rank-Tests bestimmt. Modifiziert aus: Ugurel et al. [56].

Marker für fortgeschrittene Erkrankungsstadien (AJCC III/IV), nicht jedoch für die frühen Stadien der Melanomerkrankung (AJCC I/II) dar.

\section{Diskussion und Ausblick}

Zusammenfassend lässt sich feststellen, dass eine sinnvolle Selektion hinreichend sensitiver prognostischer Serummarker für das maligne Melanom weiterführender Untersuchungen hinsichtlich der Wertigkeit und Durchführbarkeit bedarf. Möglicherweise ist für eine genauere Einschätzung der Prognose eines Patienten ein einzelner Marker nicht ausreichend, sondern die Vorhersagekraft durch die Kombination mehrerer Marker deutlich steigerbar. Insbesondere für Patienten in frühen Erkrankungsstadien vor Nachweis einer Metastasierung bedarf es hinreichend sensitiver und weitläufig einsetzbarer prognostischer Marker, um so identifizierte Risiko-Patienten weiterführenden diagnostischen und/oder therapeutischen Maßnahmen zuzuführen.

\section{Literatur}

${ }^{1}$ Swerdlow AJ. International trends in cutaneous melanoma. Ann NY Acad Sci 1990; 60: 235-251

2 McMasters KM, Reintgen DS, Ross MI et al. Sentinel lymph node biopsy for melanoma: controversy despite widespread agreement. J Clin Oncol 2001; 19: $2851-2855$

${ }^{3}$ Guo HB, Stoffel-Wagner B, Bierwirth T, Mezger J, Klingmuller D. Clinical significance of serum S100 in metastatic malignant melanoma. Eur J Cancer 1995; 31: 924-928

${ }^{4}$ Hauschild A, Engel G, Brenner W et al. S100B protein detection in serum is a significant prognostic factor in metastatic melanoma. Oncology 1999; 56: 338 - 344
${ }^{5}$ Hauschild A, Engel G, Brenner W et al. Predictive value of serum S100B for monitoring patients with metastatic melanoma during chemotherapy and/or immunotherapy. Br J Dermatol 1999; 140: 1065-1071

${ }^{6}$ Bosserhoff AK, Kaufmann M, Kaluza B et al. Melanoma-inhibitory activity, a novel serum marker for progression of malignant melanoma. Cancer Res 1997; 57: 3149-3153

${ }^{7}$ Stahlecker J, Gauger A, Bosserhoff A, Buettner R, Ring J, Hein R. MIA as a reliable tumor marker in the serum of patients with malignant melanoma. Anticancer Res 2000; 20: 5041 - 5044

${ }^{8}$ Deichmann M, Brenner A, Bock M et al. S100-beta, melanoma-inhibiting activity and lactate dehydrogenase discriminate progressive from non-progressive American Joint Committee on Cancer stage IV melanoma. J Clin Oncol 1999; 17: 1891 - 1896

${ }^{9}$ Ruiz-Cabello F, Garrido F. HLA and cancer: from research to clinical impact. Immunol Today 1998; 19: 539-542

${ }^{10}$ van Rood JJ, van Leeuwen A, van Santen MC. Anti HL-A2 inhibitor in normal human serum. Nature 1970; 226: 366-367

11 Reisfeld RA, Allison JP, Ferrone S, Pellegrino MA, Poulik MD. HL-A antigens in serum and urine: isolation, characterization, and immunogenic properties. Transplant Proc 1976; 8: $173-178$

12 Zavazava N, Kronke M. Soluble HLA class I molecules induce apoptosis in alloreactive cytotoxic T lymphocytes. Nat Med 1996; 2: 1005-1010

${ }^{13}$ Filaci G, Contini P, Brenci S et al. Increased serum concentration of soluble HLA-DR antigens in HIV infection and following transplantation. Tissue Antigens 1995; 46: 117 - 123

14 Ditschkowski M, Kreuzfelder E, Rebmann V et al. HLA-DR expression and soluble HLA-DR levels in septic patients after trauma. Ann Surg 1999; 229: 246 - 254

${ }^{15}$ Rebmann V, Ugurel S, Tilgen W, Reinhold U, Grosse-Wilde H. Soluble HLA-DR is a potent predictive indicator of disease progression in serum from early-stage melanoma patients. Int J Cancer 2002: (in press)

${ }^{16}$ Ferrone S, Marincola FM. Loss of HLA class I antigens by melanoma cells: molecular mechanisms, functional significance and clinical relevance. Immunol Today 1995; 16: 487-494

17 Westhoff U, Fox C, Otto FJ. Soluble HLA class I antigens in plasma of patients with malignant melanoma. Anticancer Res 1998; 18: $3789-3792$

${ }^{18}$ Le Bouteiller P, Blaschitz A. The functionality of HLA-G is emerging. Immunol Rev 1999; 167: 233-244

${ }^{19}$ Carosella ED, Rouas-Freiss N, Paul P, Dausset J. HLA-G: a tolerance molecule from the major histocompatibility complex. Immunol Today 1999; 20: 60-62 
${ }^{20}$ Paul P, Rouas-Freiss N, Khalil-Daher I et al. HLA-G expression in melanoma: a way for tumor cells to escape from immunsurveillance. Proc Natl Acad Sci U S A 1998; 95: 4510-4515

${ }^{21}$ Rebmann V, Pfeiffer K, Passler M et al. Detection of soluble HLA-G molecules in plasma and amniotic fluid. Tissue Antigens 1999; 53: $14-22$

22 Ugurel S, Rebmann V, Ferrone S, Tilgen W, Grosse-Wilde H, Reinhold U. Soluble human leukocyte antigen-G serum level is elevated in melanoma patients and is further increased by interferon-alpha immunotherapy. Cancer 2001; 92: 369-376

${ }^{23}$ Nagata S. Fas and Fas ligand: a death factor and its receptor. Adv Immunol 1994; 57: 129-144

${ }^{24}$ Chouaib S, Asselin-Paturel C, Mami-Chouaib F, Caignard A, Blay JY. The host-tumor immune conflict: from immunosuppression to resistance and destruction. Immunol Today 1997; 18: 493 - 497

25 Owen-Schaub LB, Radinsky R, Kruzel E, Berry K, Yonehara S. Anti-Fas on nonhematopoietic tumors: levels of Fas/APO-1 and bcl-2 are not predictive of biological responsiveness. Cancer Res 1994; 54: $1580-1586$

${ }^{26}$ O'Connell J, Bennett MW, O'Sullivan GC, Collins JK, Shanahan F. The Fas counterattack: cancer as a site of immune privilege. Immunol Today 1999; 20: 46-52

${ }^{27}$ Hahne M, Rimoldi D, Schroeter M et al. Melanoma cell expression of Fas (Apo-1/CD95) ligand: implications for tumor immune escape. Science 1996; 274: $1363-1366$

${ }^{28}$ Rivoltini L, Radrizzani M, Accornero P et al. Human melanoma-reactive CD4+ and CD8+ CTL clones resist Fas ligand-induced apoptosis and use Fas/Fas ligand-independent mechanisms for tumor killing. J Immunol 1998; 161: 1220-1230

${ }^{29}$ Thomas WD, Hersey P. CD4 T cells kill melanoma cells by mechanisms that are independent of Fas (CD95). Int J Cancer 1998; 75: 384-390

${ }^{30}$ Ugurel S, Seiter S, Rappl G, Stark A, Tilgen W, Reinhold U. Heterogenous susceptibility to CD95-induced apoptosis in melanoma cells correlates with bcl-2 and bcl-x expression and is sensitive to modulation by interferon-gamma. Int J Cancer 1999; 82: 727-736

${ }^{31}$ Raisova M, Bektas M, Wieder T et al. Resistance to CD95/Fas-induced and ceramide-mediated apoptosis of human melanoma cells is caused by a defective mitochondrial cytochrome c release. Febs Lett 2000; 473: $27-32$

32 Owen-Schaub LB, van Golen KL, Hill LL, Price JE. Fas and Fas ligand interactions suppress melanoma lung metastasis. J Exp Med 1998; 188: $1717-1723$

${ }^{33}$ Cheng J, Zhou T, Liu C et al. Protection from Fas-mediated apoptosis by a soluble form of the Fas molecule. Science 1994; 263: 1759-1762

${ }^{34}$ Cascino I, Fiucci G, Papoff G, Ruberti G. Three functional soluble forms of the human apoptosis-inducing Fas molecule are produced by alternative splicing. J Immunol 1995; 154: $2706-2713$

${ }^{35}$ Jodo S, Kobayashi S, Kayagaki $\mathrm{N}$ et al. Serum levels of soluble Fas/ APO-1 (CD95) and its molecular structure in patients with systemic lupus erythematosus (SLE) and other autoimmune diseases. Clin Exp Immunol 1997; 107: 89-95

${ }^{36}$ Midis GP, Shen Y, Owen-Schaub LB. Elevated soluble Fas (sFas) levels in nonhematopoietic human malignancy. Cancer Res 1996; 56: $3870-3874$

${ }^{37}$ Munker R, Midis G, Owen-Schaub L, Andreff M. Soluble FAS (CD95) is not elevated in the serum of patients with myeloid leukemias, myeloproliferative and myelodysplastic syndromes. Leukemia 1996; 10: $1531-1533$
${ }^{38}$ Munker R, Younes A, Cabanillas F, Andreeff M. Soluble CD95 in the serum of patients with low and intermediate grade malignant lymphomas: absence of prognostic correlations. Leuk Lymphoma 1997; 27: $517-521$

${ }^{39}$ Mizutani Y, Yoshida O, Bonavida B. Prognostic significance of soluble Fas in the serum of patients with bladder cancer. J Urol 1998; 160: $571-576$

${ }^{40}$ Jodo S, Kobayashi S, Nakajima Y et al. Elevated serum levels of soluble Fas/APO-1 (CD95) in patients with hepatocellular carcinoma. Clin Exp Immunol 1998; 112: 166-171

${ }^{41}$ Streffer JR, Schuster M, Zipp F, Weller M. Soluble CD95 (Fas/APO-1) in malignant glioma: (no) implications for CD95-based immunotherapy? J Neurooncol 1998; 40: 233-235

42 Ueno T, Toi M, Tominaga T. Circulating soluble Fas concentration in breast cancer patients. Clin Cancer Res 1999; 5: 3529-3533

${ }^{43}$ Nonomura N, Nishimura K, Ono Y et al. Soluble Fas in serum from patients with renal cell carcinoma. Urology 2000; 55: 151 - 155

${ }^{44}$ Hefler L, Mayerhofer K, Nardi A, Reinthaller A, Kainz C, Tempfer C. Serum soluble Fas levels in ovarian cancer. Obstet Gynecol 2000; 96: 65-69

${ }^{45}$ Mouawad R, Khayat D, Soubrane C. Plasma Fas ligand, an inducer of apoptosis, and plasma soluble Fas, an inhibitor of apoptosis, in advanced melanoma. Melanoma Res 2000; 10: 461 - 467

46 Ugurel S, Rappl G, Tilgen W, Reinhold U. Increased soluble CD95 (sFas/ CD95) serum level correlates with poor prognosis in melanoma patients. Clin Cancer Res 2001; 7: 1282-1286

47 Walz A, Peveri P, Aschauer AO, Baggiolini M. Purification and amino acid sequencing of NAF, a novel neutrophil activating factor produced by monocytes. Biochem Biophys Res Commun 1987; 149: 755 - 761

${ }^{48}$ Koch AE, Polverini PJ, Kunkel SL et al. Interleukin-8 as a macrophage derived mediator of angiogenesis. Science 1992; 258: 1798-1801

${ }^{49}$ Schadendorf D, Möller A, Algemissen B, Worm M, Sticherling M, Czarnetzki BM. IL-8 produced by human malignant melanoma cells in vitro is an autocrine growth factor. J Immunol 1993; 151: 2667-2675

50 Wang JM, Taroboletti G, Matsushima K, Damme JV, Mantovani A. Induction of haptotactic migration of melanoma cells by neutrophil activating protein/IL-8. Biochem Biophys Res Commun 1990; 169: $165-170$

51 Singh RK, Gutman M, Radinsky R, Bucana CD, Fiedler IJ. Expression of interleukin-8 correlates with metastatic potential of human melanoma cells in nude mice. Cancer Res 1994; 54: 3242 - 3247

52 Singh RK, Gutman M, Reich R, Bar-Eli M. Ultraviolet B irradiation promotes tumorigenic and metastatic properties in primary cutaneous melanoma via induction of interleukin-8. Cancer Res 1995; 55: $3669-3674$

${ }^{53}$ Luca M, Huang S, Gershenwald JE, Singh RK, Reich R, Bar-Eli M. Expression of interleukin-8 by human melanoma cells up-regulates MMP-2 activity and increases tumor growth and metastasis. Am J Pathol 1997; 151: $1105-1113$

${ }^{54}$ Nürnberg W, Tobias D, Otto F, Henz BM, Schadendorf D. Expression of interleukin-8 detected by in situ hybridization correlates with worse prognosis in primary cutaneous melanoma. J Pathol 1999; 189: 546-551

${ }^{55}$ Scheibenbogen C, Mohler T, Haefele J, Hunstein W, Keilholz U. Serum interleukin-8 (IL-8) is elevated in patients with metastatic melanoma and correlates with tumor load. Melanoma Res 1995; 5: 179-181

${ }^{56}$ Ugurel S, Rappl G, Tilgen W, Reinhold U. Increased serum concentration of angiogenic factors in malignant melanoma patients correlates with tumor progression and survival. J Clin Oncol 2001; 19: 577-583 